\title{
Subwavelength Acoustic Vortex Beams Using Self-Demodulation
}

\author{
Noé Jiménez®, ${ }^{1, *}$ Joao Ealo®, ${ }^{2}$ Rubén D. Muelas-Hurtado® ${ }^{2,3}$ Aroune Duclos, ${ }^{4}$ and \\ Vicent Romero-García $\oplus^{4}$ \\ ${ }^{1}$ Instituto de Instrumentación para Imagen Molecular (I3M), Universitat Politècnica de València-CSIC, \\ València 46022, Spain \\ ${ }^{2}$ School of Mechanical Engineering, Universidad del Valle, Cali 760032, Colombia \\ ${ }^{3}$ School of Civil and Geomatic Engineering, Universidad del Valle, Cali 760032, Colombia \\ ${ }^{4}$ Laboratoire d'Acoustique de l'Université du Mans (LAUM), UMR CNRS 6613, Institut d'Acoustique-Graduate \\ School (IA-GS), CNRS, Le Mans Université, 72085 Le Mans cedex 9, France
}

(Received 29 October 2020; revised 16 March 2021; accepted 16 April 2021; published 13 May 2021)

\begin{abstract}
Acoustic vortices with subwavelength dimensions and tunable topological charge are theoretically and experimentally synthesized at distances far beyond the Rayleigh diffraction length of the source, using self-demodulation. A dual helical acoustic source is used to generate two primary confocal vortex beams at different frequencies and different topological charges. As a consequence of the conservation of angular momentum during nonlinear wave mixing, a self-demodulated vortex beam at the difference frequency emerges, keeping the spatial features of the primary vortex beams and a topological charge that is the difference of their topological charges. We report subdiffractive vortices the characteristic size of which is 18 times smaller than its wavelength at a distance 2.8 times the Rayleigh diffraction length. The generation and focusing of subwavelength vortices paves the way for long-range communication, biomedical, and wave-matter interaction applications.
\end{abstract}

DOI: 10.1103/PhysRevApplied.15.054027

\section{INTRODUCTION}

The transfer of energy and momentum from acoustic and elastic waves to matter is currently being used to develop ground-breaking applications. Novel wave-matter interaction mechanisms have allowed emerging applications, e.g., the recent advances in neuroscience using thermal ablation of deep-brain nuclei with submillimetric precision [1], the mechanical interaction of focused ultrasound beams with tissues for noninvasive and localized neuromodulation [2], or targeted drug delivery in the brain [3]. Interestingly, in addition to linear momentum, wave fields with spiral phase dislocations, i.e., acoustic vortices [4], carry orbital angular momentum. These acoustic vortices can transfer their angular momentum to solid objects and exert mechanical torques [5-8].

Vortex beams are characterized by a collimated wave front with a phase dependence of the type $e^{i l \phi}$, where $\phi$ is the azimuthal angle and $l$ is the topological charge, respectively. At the principal axis of the vortex beam, the field exhibits a phase singularity and the field is null. These singular fields can produce negative radiation forces on small particles, offering unique possibilities for manipulating matter [9]. Recently, they have received increasing

\footnotetext{
*nojigon@upv.es
}

interest mainly due to direct practical applications for particle trapping and manipulation [10-15]. Acoustic vortices can exert mechanical forces several orders of magnitude higher than those of their optical counterparts $[16,17]$ with reduced thermal effects. They have recently shown potential applications to the manipulation of individual cells without inducing photothermal and/or photochemical damage [18], to trapping and guiding kidney-stone fragments [19], or to selectively guiding individual drugdelivery carriers based on microbubbles [20].

In order to generate a vortex beam, precise control of the acoustic field is required. Methods to synthesize these beams include active devices such as phased arrays [21-24], helical radiating surfaces [21,25], spiral diffraction gratings following an Archimedes' spiral [26-28] to generate high-order Bessel beams, Fresnel-spiral diffraction gratings [29] to generate sharply focused vortex beams, diverging [30] and converging (see the Supplemental Material in Ref. [29]) Archimedean spirals, or active vibrating surfaces with a spiral shape [31,32]. One version of these active spirals takes the form of interdigitated transducers [33], the spiraling shape of which can encode the phase of the field like a hologram and generate vortices through the generation of swirling Rayleigh waves [34]. The whole holographic information of a vortex can be encoded in an acoustic hologram, enabling the 
generation of high-order Bessel beams with a long depth of field in homogeneous media [35]. Acoustic holograms can also generate vortex beams through complex biological media, e.g., correction of the phase aberrations during transcranial propagation [36]. Acoustic vortices can also be generated by systems that exploit the photoacoustic effect [37]. Recently, metamaterials have been used to generate vortex beams by using flat and subwavelength structures [38-42], allowing precise manipulation of the transmitted phase by tuning the geometry of the resonators at selected frequencies.

However, in all these configurations, the natural diffraction of the wave front limits the size of the vortex. For example, a high-order Bessel beam, the transversal pressure profile of which is described by a Bessel function of the first kind and order $l, p(r, \phi)=J_{l}\left(k_{r} r\right) \exp (i l \phi)$, presents a minimum distance between pressure maxima of $D_{\min } \approx \lambda\left(|l|+0.8086|l|^{1 / 3}\right) / \pi$, where $\lambda$ is the wavelength [27]. In this case, diffraction limits the minimum size of such vortices to $D_{\min } \approx 0.57 \lambda$ for $l=1$ and this value is only reached for distances smaller than the Rayleigh diffraction length of the source due to the divergence of the beam, given by $S / \lambda$, where $S$ is the surface of a circularaperture source. Recently, increasing attention has been paid to restoring the evanescent components of a field to overcome the diffraction limit using either metamaterials [43-45] or time-reversal techniques [46]. However, as these approaches rely on evanescent waves, their use is restricted to near-field distances.

The synthesis of a vortex of small size at low frequency can be of great interest to many practical applications. For example, in underwater communications using acoustic vortex transceivers [47-50], the size of the instrumentation for detection is of the order of the radius of the vortex. On the one hand, a mechanism to reduce the width of the vortex impinging on the detector should enhance the detected signal and improve the system sensibility and its performance [47]. On the other, when propagating at long distances in underwater applications, or in strongly scattering and absorbing media such as the ocean floor, ultrasonic beams are strongly attenuated. In these cases, it is desirable to emit at low ultrasonic frequencies. However, by directly reducing the frequency of the ultrasonic source, its directivity becomes very wide and, in addition, it also has a negative impact on the source efficiency because the radiation impedance decreases. A common solution is to use the self-demodulation effect to generate a parametric acoustic antenna [51]. These systems use two primary ultrasonic transducers radiating two overlapping narrow beams of high amplitude and different frequencies. The nonlinear mixing of the primary beams generates a new component at the difference frequency. This low-frequency beam is generated progressively along a long path and its radiation pattern is similar to an endfire array; it interferes destructively at the transverse direction and constructively in the forward direction. Therefore, the self-demodulated beam conserves the directivity of the primary beams [52]. Parametric beams are commercially available and used for underwater communications, sub-bottom profilers, or the detection of buried targets. In this way, obtaining a parametric vortex antenna will be desirable for applications based on vortices, such as underwater communications [47-50], propagation in inhomogeneous media [53], or imaging techniques based on vortices [54,55].

Another application that should benefit from subwavelength vortices is particle trapping in biomedical ultrasound. It has recently been demonstrated that bubbles can be trapped using ultrasound beams and that their payload can be delivered by exploiting its cavitation activity, the latter linked to the bubble resonance $[20,56]$. Many drugdelivery carriers are based on coated microbubbles of a small size, of the order of several micrometers [57]. In this way, a mechanism to control the trapping size, linked to the vortex diameter, and simultaneously tuning the excitation frequency, linked to the microbubble resonance, can be of great interest in the design of accurate drug-delivery applications. In addition, low-frequency vortices are more robust to phase aberrations when propagating in strongly refracting and absorbing tissues such as the bones of the skull $[36,58]$.

The task of obtaining vortices of tunable topological charge using nonlinearity has been demonstrated in optics, e.g., by second-harmonic generation using the nonlinear mixing of two beams and synthesized gratings [59] or frequency doubling using a nonlinear crystal [60], where a parametric frequency converter has been demonstrated for vortex charge doubling. Later, sum-frequency generation has been demonstrated using a nonlinear crystal and computer-generated holograms [61,62]. The frequency conversion has enabled the realization of several light processors, including arithmetical operations with the topological charges of the interacting vortices. Later, these concepts have been studied in acoustics to synthesize vortices as a result of harmonic generation by frequency doubling $[22,23]$ and by the nonlinear mixing of two vortices [63], where the laws of conservation of orbital angular momentum have also been provided. These studies have been extended to nonlinear shock waves [64] and, recently, fractional vortices have been identified in short pulsed helicoidal beams [65].

In this work, we synthesize a vortex beam of subwavelength size at a distance beyond the Rayleigh diffraction length using the nonlinear mixing of two confocal, highfrequency, and detuned vortex beams of different topological charges. We demonstrate the concept using the natural self-demodulation phenomenon in air. We generate a focused vortex beam of integer topological charge, the distance between magnitude maxima of which is approximately 18 times smaller than its wavelength at a 
distance approximately 2.8 times the Rayleigh diffraction length.

\section{METHODS}

A device is manufactured by disposing 10-mm-diameter ultrasound emitters operating in air over two different helical surfaces, as shown in Figs. 1(a) and 1(b). The helical profile of the $n$th surface (with $n=1,2$ ) is designed to generate a focused beam at a focal point $\mathbf{r}(\phi, r, z)=(0,0, F)$ and, simultaneously, produce at this location a time-offlight difference equal to $\Delta t_{n}=l_{n} \phi / \omega_{n}$, where $l_{n}$ is the topological charge of the $n$th primary vortex beam, $\omega_{n}$ is the angular frequency, and $\phi$ is the azimuthal coordinate [66]. In particular, the first source, composed of the two internal concentric rings of transducers [see Fig. 1(b)], is excited with a sinusoidal pulse burst of $\omega_{1} / 2 \pi=40$ $\mathrm{kHz}$, while the second one, composed of the two external concentric rings of transducers, is excited at $\omega_{2} / 2 \pi=41$ $\mathrm{kHz}$. The topological charges of the primary vortex beams, defined by the curvature of the helical surfaces, are set to $l_{1}=1$ and $l_{2}=2$. The source aperture is set to $2 a=110$ $\mathrm{mm}$, where $a$ is the radius of the source, and the focal point is $F=100 \mathrm{~mm}$. Thus, the Rayleigh distances for the primary vortex beams are around $\pi a^{2} / \lambda_{1} \approx \pi a^{2} / \lambda_{2} \approx 1.1 \mathrm{~m}$.

For finite-amplitude waves and when all emitters are active, both beams interact and due to material and advective nonlinearity, wave mixing occurs during propagation. Higher harmonics arise as arithmetical combinations of the fundamental waves of both beams, as is shown in the spectrum plotted in Fig. 1(c), acquired at the focal spot. The root-mean-square pressure measured is $138.4 \mathrm{~Pa}$ at 40 $\mathrm{kHz}$ (a sound pressure level of $136.8 \mathrm{~dB}$ referenced at 20 $\mu \mathrm{Pa}$ ), enough to trigger weak nonlinear effects. In addition to higher harmonics, a difference-frequency mode of frequency $\omega_{d}=\omega_{2}-\omega_{1}$ is generated due to the nonlinear self-demodulation of the beating wave front, as shown in Fig. 1(d).

As homogeneous acoustic media lack strong dispersion, phase matching holds during nonlinear propagation and harmonic generation processes are cumulative with distance. On the one hand, it is expected that the locally generated self-demodulated mode presents a field spatial distribution similar to those of the primary beams, as occurs in parametric acoustic antennas [51]. On the other hand, the phase of the self-demodulated beam depends on the spatiotemporal interference of the two vortex beams, which is linked to their topological charges [67]. This results in a self-demodulated beam with a phase factor $\exp \left(i l_{d} \phi\right)$, where the topological charge of the selfdemodulated beam is

$$
l_{d}=s_{d}\left(l_{2}-l_{1}\right)
$$

where $s_{d}=\operatorname{sign}\left(\omega_{2}-\omega_{1}\right)$, due to the conservation of topological charge of nonlinear vortices, which is indeed a consequence of the conservation of angular momentum [22]. Particular attention should be paid to Eq. (1), because this is not valid if the frequencies of the
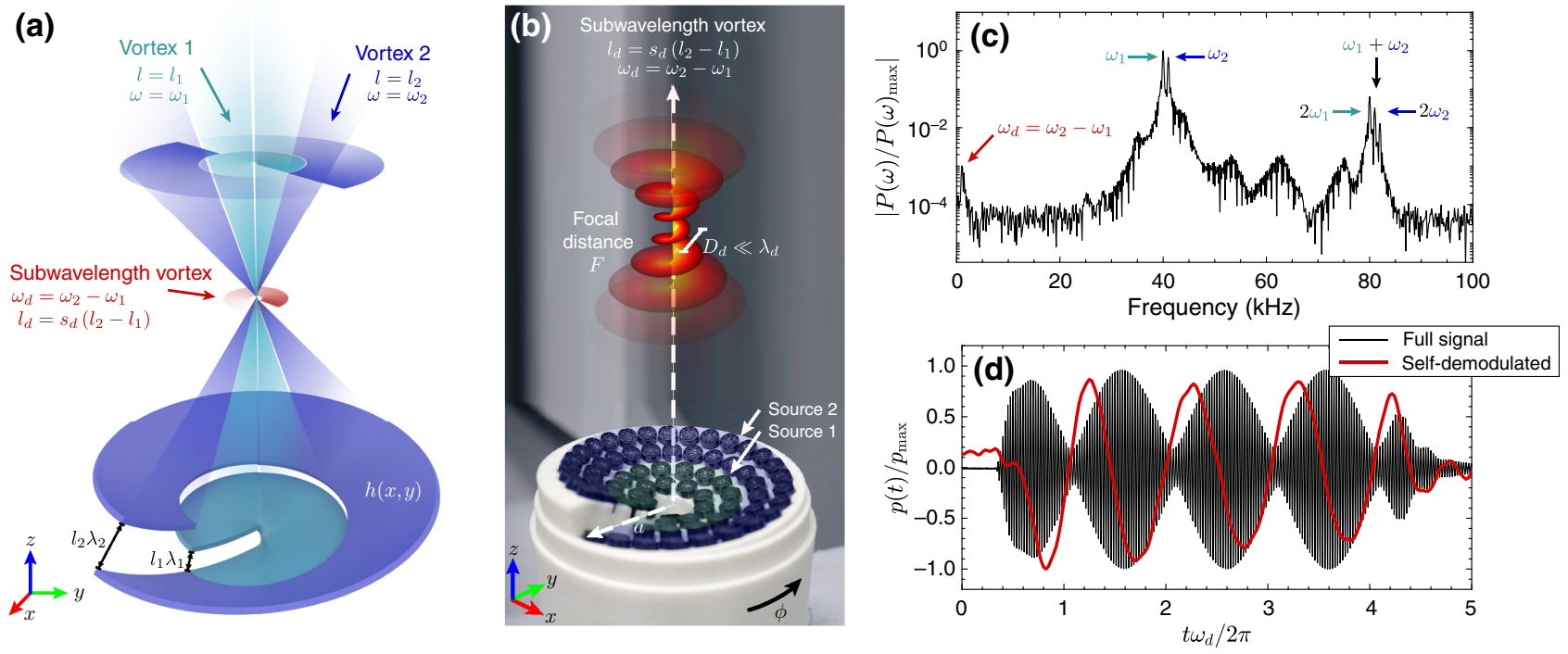

FIG. 1. (a) A schematic of the system, composed of two confocal focused vortex beams at frequencies $\omega_{1}$ and $\omega_{2}$, with topological charges $l_{1}$ and $l_{2}$, respectively. Self-demodulation is generated by nonlinearity, producing a vortex at the difference frequency $\omega_{d}=$ $\omega_{2}-\omega_{1}$, with a topological charge of $l_{d}=l_{2}-l_{1}$ and a beam width given by $D_{d}$ in the subwavelength regime. (b) A photograph of the helical source used in the experiments. (c) The measured spectrum at the focal spot, showing the nonlinear generated harmonics by combination of the two primary vortex beams. (d) The corresponding temporal waveform and low-pass filtered signal (normalized). The maximum root-mean-square pressure is $138.4 \mathrm{~Pa}$ at $40 \mathrm{kHz}$. 
primary beams are commensurable [63]. However, in selfdemodulation applications, this is usually fulfilled, as the primary beams are commonly chosen with nearby frequencies. Therefore, to synthesize a subwavelength vortex by self-demodulation, the primary beams should present different topological charges and frequencies and, in addition, their field distributions must overlap in space.

\section{RESULTS}

The field generated by each of the two confocal nonlinear vortices is shown in Fig. 2. First, the acoustic field radiated by such a device in the linear regime is theoretically obtained using the Rayleigh-Sommerfeld diffraction integral [68]. The results for small-amplitude waves, presented in Figs. 2(a)-2(c), show that the helical source correctly generates the focused vortex beams at frequencies $\omega_{1}$ and $\omega_{2}$. However, if the source is excited at $\omega_{d}$, as $k_{d} a \ll 1$, the value of the Rayleigh diffraction length is very short and the field decays rapidly due to spherical spreading.

\section{A. Nonlinear vortex generation}

A simulation is performed by solving the nonlinear Westervelt's equation [51] in a retarded coordinate frame using a spectral operator-splitting method and including thermoviscous absorption [69]. This method allows the numerical integration of wide-aperture sources beyond the parabolic approximation. The boundary conditions are calculated by integrating the field radiated by each of the transducers of the helical phased-array source over a plane located at $z=3 \mathrm{~cm}$ using the linear Rayleigh-Sommerfeld integral [70]. Then, nonlinear effects are accounted for and the field is integrated along the axial coordinate. In addition, experiments are conducted in a semianechoic environment. The emitters on the helical surfaces are excited with sinusoidal pulse-burst signals of length $t=8 \pi / \omega_{d}$ and the field is scanned by a pinhole microphone using a three-dimensional (3D) positioning system. The simulated and experimental results are shown in Figs. 2(d)-2(f) and Figs. 2(g)-2(i), respectively. First, due to weakly nonlinear propagation, the nonlinear simulated field of each of the primary vortex beams [Figs. 2(d) and 2(e)] agrees with the theoretical linearly diffracted pattern [Figs. 2(a) and 2(b)]. The corresponding experimental fields [Figs. 2(g) and 2(h)] agree with the theoretical ones. The weakly oscillating pattern in the axial direction indicates the presence of a weak counterpropagating mode caused by reflections on the positioning system. We note that the location of the pressure maxima does not correspond to the focus due to strong diffraction effects of the small-aperture source.
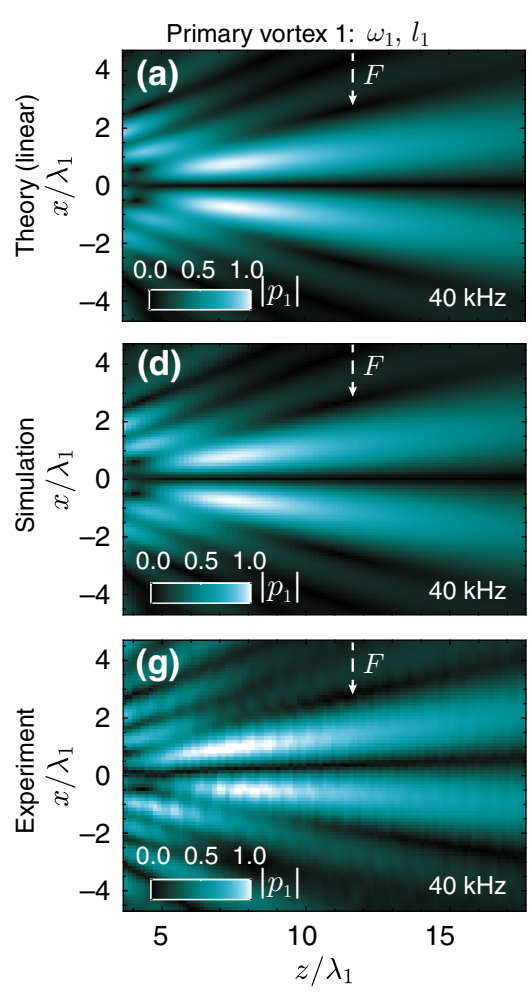

Primary vortex $2: \omega_{2}, l_{2}$
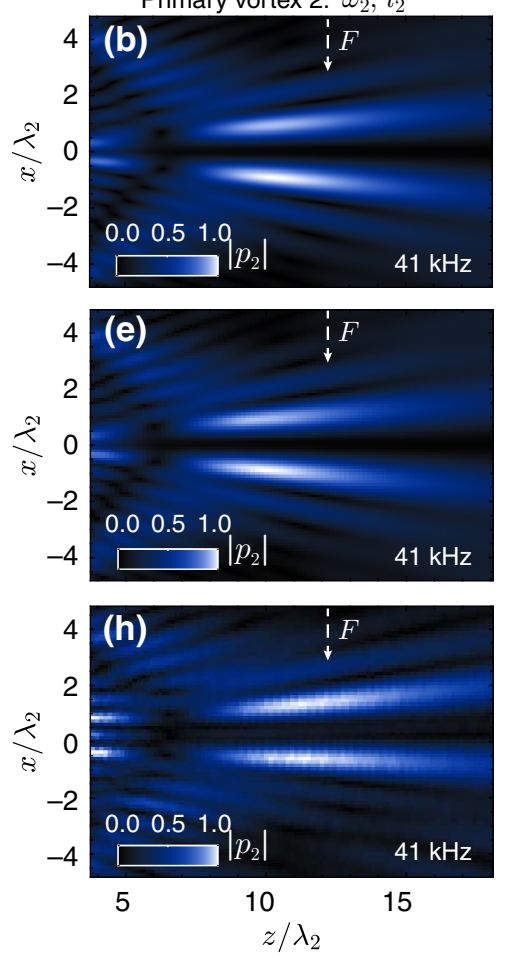
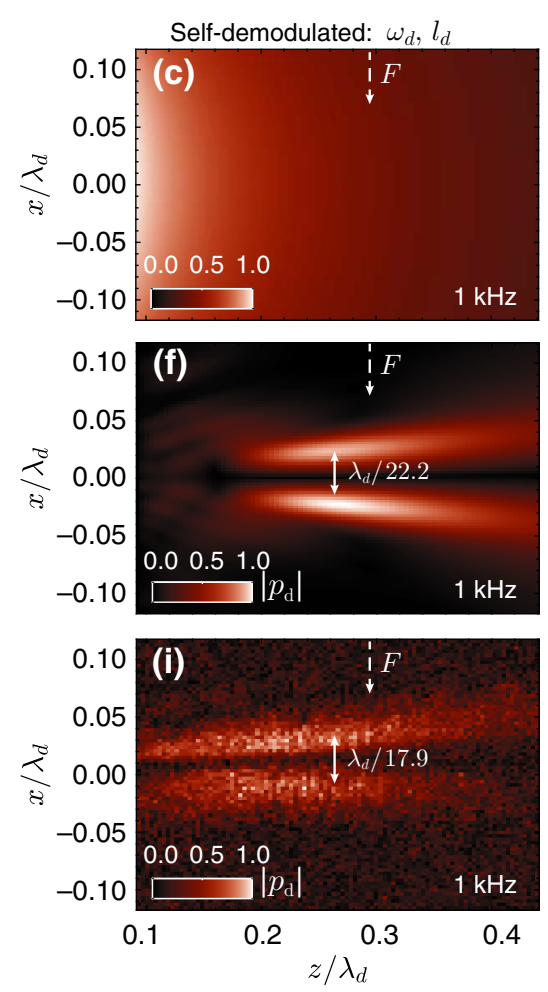

FIG. 2. A sagittal field cross section at $y=0$, obtained by (a)-(c) linear theory, (d)-(f) nonlinear simulation, and (g)-(i) experiment, showing the magnitude of the acoustic field at frequencies of $40 \mathrm{kHz}, 41 \mathrm{kHz}$, and $1 \mathrm{kHz}$, respectively. The maximum root-mean-square pressure is $138.4 \mathrm{~Pa}$ at $40 \mathrm{kHz}$. 
Primary vortex $1: \omega_{1}, l_{1}$
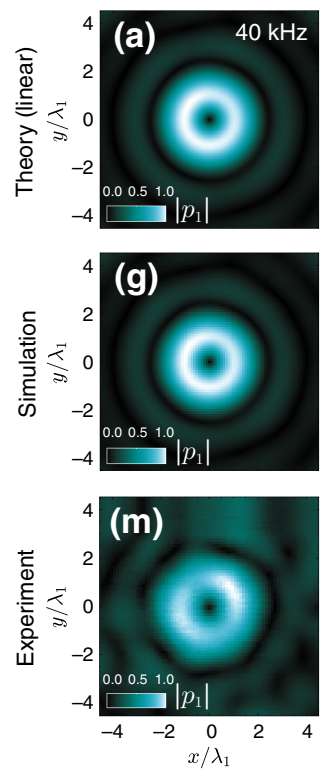
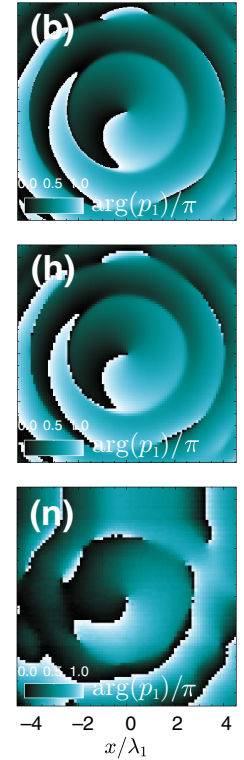

Primary vortex $2: \omega_{2}, l_{2}$
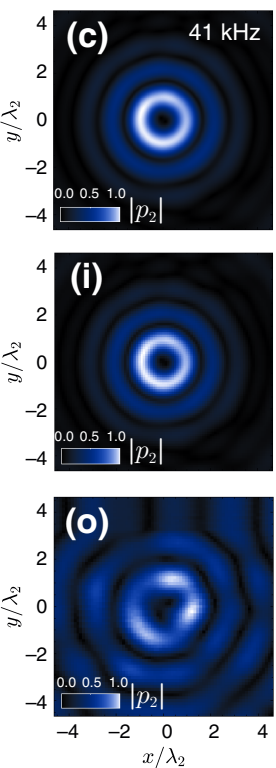
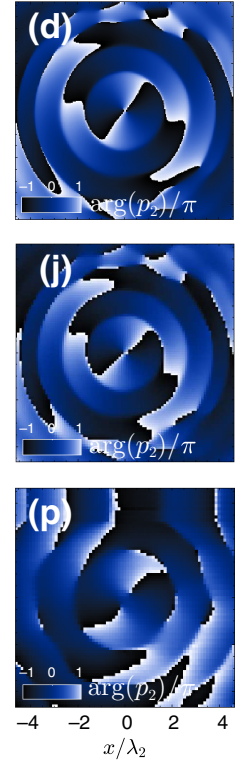

Self-demodulated: $\omega_{d}, l_{d}$
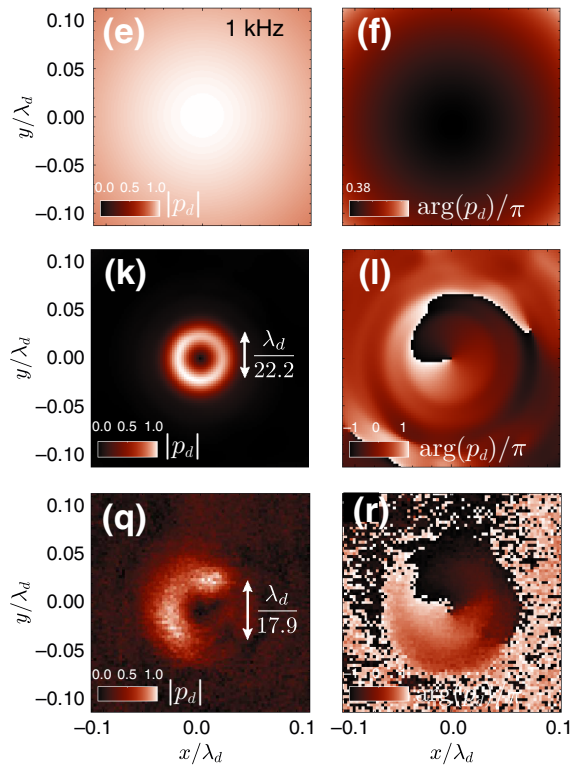

FIG. 3. A transverse-field cross section at $z=80 \mathrm{~mm}$, obtained by (a)-(f) linear theory, (g)-(l) nonlinear simulation, and (m)-(r) experiment, showing the normalized magnitude $\left(\left|p_{1}\right|\right)$ and the normalized phase $\left(\arg \left(p_{1}\right) / \pi\right)$ of the acoustic field at frequencies of $40 \mathrm{kHz}, 41 \mathrm{kHz}$, and $1 \mathrm{kHz}$, respectively. The maximum root-mean-square pressure is $138.4 \mathrm{~Pa}$ at $40 \mathrm{kHz}$.

In addition, the self-demodulated components for the simulation and experiment are shown in Figs. 2(f) and 2(i), respectively. A low-frequency beam at $\omega_{d}=1 \mathrm{kHz}$ is generated locally and its spatial distribution matches the overlapping volume of the two primary beams. Its corresponding wavelength is $\lambda_{d}=2 \pi c_{0} / \omega_{d} \gg \lambda_{n}$; therefore, the width of the beam, dominated by the width of the primary beams, is deep subwavelength.

\section{B. Subdiffractive vortices}

The transversal cross sections at the plane $\mathbf{r}(x, y, z)=$ $(x, y, F)$ corresponding to the cases analysed in Fig. 2 are
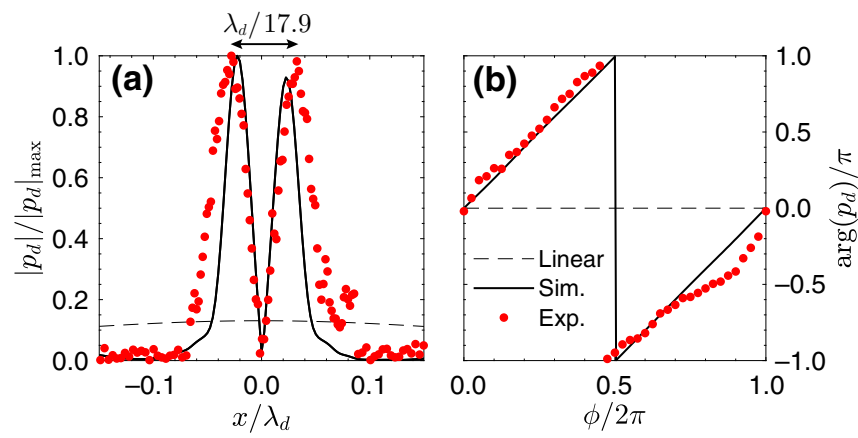

FIG. 4. (a) The normalized field-magnitude cross section $p(x, 0,80 \mathrm{~mm})$ at $1 \mathrm{kHz}$ (difference frequency) obtained experimentally (markers), numerically (continuous), and by linear theory (dashed). (b) The phase of the self-demodulated field along the azimuthal coordinate. shown in Fig. 3. First, the theoretical field distributions of the primary beams calculated in the linear regime are shown in Figs. 3(a)-(d). The helical source correctly generates a focused vortex with topological charge $l_{1}=1$ and $l_{2}=2$, respectively, and the field vanishes at the phase singularity. However, the linear propagation of a $1-\mathrm{kHz}$ wave does not present such a phase dislocation [Figs. 3(e) and 3(f)]. In addition, as $F$ is much larger than the Rayleigh diffraction length for $1 \mathrm{kHz}, S / \lambda_{d} \approx F / 3.6=$ $2.8 \mathrm{~cm}$, strong diffraction dominates the propagation and this low-frequency beam cannot be collimated.

In the nonlinear regime, the magnitude and the phase distributions of the two primary vortex beams, shown in Figs. 3(g)-3(j) and 3(m)-3(p) for simulations and measurements, respectively, also confirm the formation of a focused helical beam. The simulations and experiments are in good agreement; only small discrepancies are observed for transverse pattern of the $41-\mathrm{kHz}$ primary beam in the measurements. The self-demodulated vortex arises as a result of the nonlinear mixing of the two primary vortex beams [Figs. 3(k) and 3(l) and 3(q) and 3(r) for simulations and experiments, respectively]. Its topological charge is given by $l_{d}=l_{2}-l_{1}=1$, showing the conservation of topological charge and, therefore, the conservation of orbital angular momentum during the nonlinear mixing of the primary beams as given by Eq. (1).

Figure 4 shows the transversal pressure-field distribution of the self-demodulated vortex beam at $z=80 \mathrm{~mm}$ and $y=0 \mathrm{~mm}$. The width of the vortex, $D_{d}$, is 17.9 times smaller than the wavelength in the experimental 
observations ( $D_{d}=\lambda_{d} / 22.2$ in the simulations). Note that the measurement distance is 2.8 times the Rayleigh diffraction length for the self-demodulated mode. The field at the axis $(x=0)$ becomes null due to the phase singularity. The phase of the beam along the azimuthal coordinate $\phi$, depicted in Fig. 4(b), agrees with a linear profile of $\left(l_{2}-\right.$ $\left.l_{1}\right) \phi=l_{d} \phi$, demonstrating the topological-charge conservation during nonlinear mixing. Using this approach, vortices of arbitrary topological charge and size can be synthesized by tuning the parameters of the primary beams [71].

\section{CONCLUSIONS}

In this work, we show the subwavelength and subdiffractive generation of acoustic vortices at distances beyond the Rayleigh diffraction length by using nonlinear selfdemodulation. To generate vortices below the diffraction limit by self-demodulation, the primary vortex beams should present different topological charges and frequencies and their field distributions must overlap in space. Subwavelength vortices emerge as a result of the spatiotemporal interference of two primary vortex beams due to the conservation of angular momentum during nonlinear wave mixing.

The synthesis of vortices below the diffraction limit is of great interest for many applications such as underwater acoustic communications using vortex transceivers [47]. As occurs in parametric acoustic antennas, subwavelength vortices are generated all along the endfire virtual array formed by the primary beams. Therefore, self-demodulated vortices should conserve the radiation pattern of the primary beams because in the transverse direction they interfere destructively. However, the energy is transmitted at the difference frequency. For this reason, vortex-encoded information using self-demodulated vortex beams can be transmitted at higher distances due to lower absorption and scattering when traveling in complex media.

Moreover, using standard vortices, the trapping size is linked to the frequency of the beam and the topological charge. By self-demodulation, the size of the vortex can be tuned by the primary frequencies, while the frequency is a free parameter that depends on the detuning of the primary beams. This mechanism enables a degree of freedom to control and manipulate resonating particles such as bubbles or coated microbubbles. In this case, the trap allows the manipulation, while by tuning the difference frequency one can control the cavitation activity, the latter linked by the resonance of the target. In the context of biomedical ultrasound applications, controlling the cavitation activity of trapped microbubbles is useful in the design of drug-delivery carriers to deliver the payload of the resonating therapeutic agent. Miniaturization [72] of the proposed concept can be performed using helical surfaces, holograms, spiral diffraction gratings, or a pair of coiled spiral sources.

These results show that vortices of arbitrary topological charge with tunable and subwavelength width can be generated using self-demodulation. This work may pave the way for practical applications such as long-range underwater communication systems based on vortex transceivers or the trapping and manipulation of particles and microbubbles for biomedical ultrasound applications.

\section{ACKNOWLEDGMENTS}

This research has been supported by the Spanish Ministry of Science, Innovation and Universities through "Juan de la Cierva-Incorporación" Grants No. IJC2018037897-I and No. PID2019-111436RB-C22, by the Agència Valenciana de la Innovació through Grants No. INNVA1/2020/92 and No. INNCON/2021/8, and by the HYPERMETA project, funded under the "Etoiles Montantes" program of the Région Pays de la Loire. R.D.M.-H acknowledges support from Colciencias Scholarship Program No. 727-2015.

\section{APPENDIX A: HELICAL SOURCE DESIGN}

The generation of the two confocal primary vortex beams is based on a helical surface. The surface should produce the focusing of the acoustic waves at the focal point $\mathbf{r}(x, y, z)=\mathbf{r}(0,0, F)$ and, simultaneously, a phase dislocation at this point. The time of flight at the focal spot should present an azimuthal dependence of the type

$$
\Delta t_{n}(\phi)=\frac{l_{n} \phi}{\omega_{n}}
$$

where $l_{n}$ is the topological charge, $\omega_{n}$ is the angular frequency of the $n$th vortex beam, and $\phi$ is the azimuthal angle. To guarantee such a phase, we design a helical surface as shown in Fig. 5. The surface design is constrained by the distance to the focal point, which must follow the relation

$$
R_{n}(\phi)=F-\frac{l_{n} \lambda_{n} \phi}{2 \pi}
$$

at any point, where $\lambda_{n}=2 \pi c_{0} / \omega_{n}$. This surface, defined in spherical coordinates as $\mathbf{r}=\mathbf{r}\left[\phi, \theta, R_{n}(\phi)\right]$, can be expressed in Cartesian coordinates as

$$
\begin{aligned}
& x_{n}(\phi, \theta)=R_{n}(\phi) \cos (\phi) \sin (\theta), \\
& y_{n}(\phi, \theta)=R_{n}(\phi) \sin (\phi) \sin (\theta), \\
& z_{n}(\phi, \theta)=-\left[F+R_{n}(\phi) \cos (\theta)\right],
\end{aligned}
$$




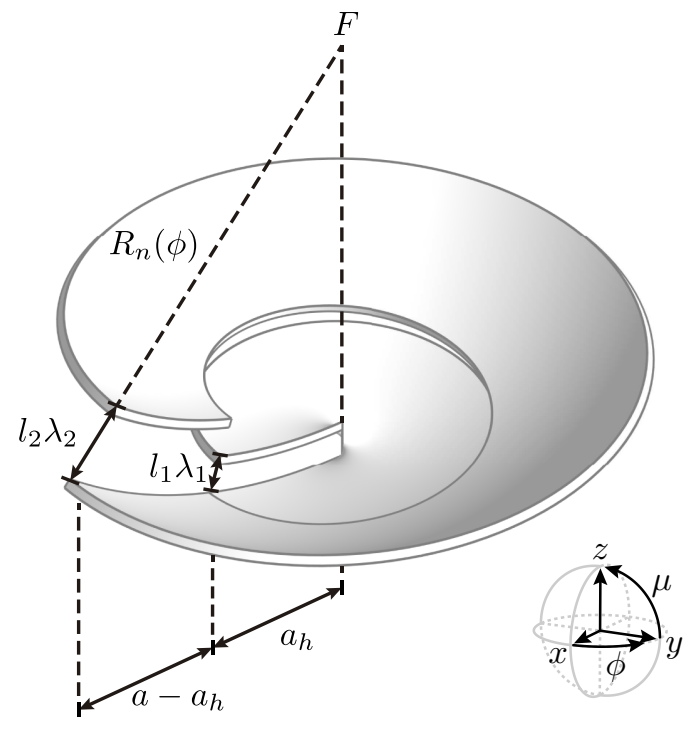

FIG. 5. A schematic of the helical surface to focus two confocal vortex beams.

where the azimuthal and elevation angles range between $0<\phi<2 \pi$ and $\theta_{\min }^{[n]}<\theta<\theta_{\max }^{[n]}$, respectively. The aperture angles are given by

$$
\begin{aligned}
\theta_{\max }^{[2]} & =\tan ^{-1}\left(\frac{a}{2 F}\right), \\
\theta_{\min }^{[2]} & =\tan ^{-1}\left(\frac{a_{h}}{2 F}\right), \\
\theta_{\max }^{[1]} & =\theta_{\min }^{[2]}, \\
\theta_{\min }^{[1]} & =0,
\end{aligned}
$$

where $2 a$ is the outer-source aperture and $2 a_{h}$ is the aperture of the inner source.

\section{APPENDIX B: EXPERIMENTAL METHODS}

The two vortex sources are manufactured by placing two concentric arrays of transducers over the helical surfaces. Each of the sources is composed of two concentric arrays of piezoelectric transducers, as shown in Fig. 6. Each piezoelectric transducer (MA40S4S, Murata Manufacturing Co., Ltd.) presents an aperture of $10 \mathrm{~mm}$ and produces a sound pressure level of $120 \mathrm{~dB}$ SPL $(0 \mathrm{~dB}=$ $20 \mu \mathrm{Pa}$ ) measured at $30 \mathrm{~cm}$ in air when excited with a sinusoidal signal of $10 \mathrm{~V}$. The source consists of a total of 74 transducers ( 50 for the inner and 24 for the outer source). The transducers are connected in parallel. For the first source (inner array), the design frequency is $f_{1}=40$ $\mathrm{kHz}$, while for the second one (outer array), the frequency is $f_{2}=41 \mathrm{kHz}$. The topological charges of the primary vortex beams are set to $l_{1}=1$ and $l_{2}=2$. The source aperture is $2 a=110 \mathrm{~mm}\left(2 a_{h}=60 \mathrm{~mm}\right)$ and the focal point is $F=100 \mathrm{~mm}$.
Signals are generated using an arbitrary function generator (AFG3022B, Tektronix, Capitol, United Kingdom) and amplified by a $60-\mathrm{dB}$ gain $100-\mathrm{W}$ power amplifier (Type 2713, Bruel \& Kjaer, Naerum, Denmark). Each array is excited with a sinusoidal pulse burst of $4 \mathrm{~ms}$ duration. To guarantee a similar amplitude at the focal point, the inner array and the outer array are excited with different amplitudes, of $10 \mathrm{~V}$ and $12 \mathrm{~V}$, respectively. This difference is likely due to the different number of transducers in each array presenting different impedances to the two amplifiers. These values are adjusted experimentally. The measurements are conducted in a semianechoic environment. The acoustic signals are acquired by using a 1/4-in. pressure-field microphone (G.R.A.S. Holte, Denmark) with a preamplifier (Type 26TC, G.R.A.S. Holte, Denmark) and a signal-conditioning module (12AQ, G.R.A.S. Holte, Denmark), and using a custom 2-mm-diameter pinhole. The signals are acquired using a digitizer (Picoscope 5244B) with a sample rate of $6.5 \mathrm{MHz}$. A total of 20 averages per measurement are performed to reduce noise.

The effect of the pinhole is evaluated experimentally by measuring the amplitude of an acoustic source at the axial direction with and without using the pinhole. Using the ultrasonic transducer as the source, we measure an attenuation of $18.7 \mathrm{~dB}$ for $40 \mathrm{kHz}$, and $19.15 \mathrm{~dB}$ for $41 \mathrm{kHz}$. Using a commercial electrodynamic loudspeaker, the measured attenuation is $0.026 \mathrm{~dB}$ at $1 \mathrm{kHz}$. At high frequencies, the effect of the pinhole is important in terms of the measured amplitude, reducing the amplitude of the detected ultrasonic wave. However, at the self-demodulation frequency, the sensibility of the microphone is not strongly affected, while the pinhole allows us to scan the field with high spatial resolution.

The field is scanned using a 3D positioning system (Mistras NDT automation 1606). For the sagittal scans, $p=$ $p(x, 0, z)$, the space is scanned using steps of $0.1 \mathrm{~mm}$ in the range $30<z<180 \mathrm{~mm}$ and $-20<x<20 \mathrm{~mm}$. For the transversal scans, $p=p\left(x, y, z_{m}\right)$, the space is scanned using steps of $0.1 \mathrm{~mm}$ in the range $-20<x<20 \mathrm{~mm}$ and $-20<y<20 \mathrm{~mm}$, at a height $z_{m}=90 \mathrm{~mm}$ that corresponds to the pressure maxima of the field. We note that the location of the pressure maxima does not correspond to the focus $\left(z_{m}<F\right)$ due to wave diffraction.

Band-pass filtering is applied to show the demodulated signal in the time domain by using a zero-gain zero-phase peak-filter. For the pressure-field distributions, the field amplitude at each component is calculated using a fast Fourier transform.

\section{APPENDIX C: NUMERICAL METHODS}

\section{Linear regime: Rayleigh-Sommerfeld integral}

The acoustic field at a point $\mathbf{r}$, generated by the radiating sources located along the helical surface, is obtained using 


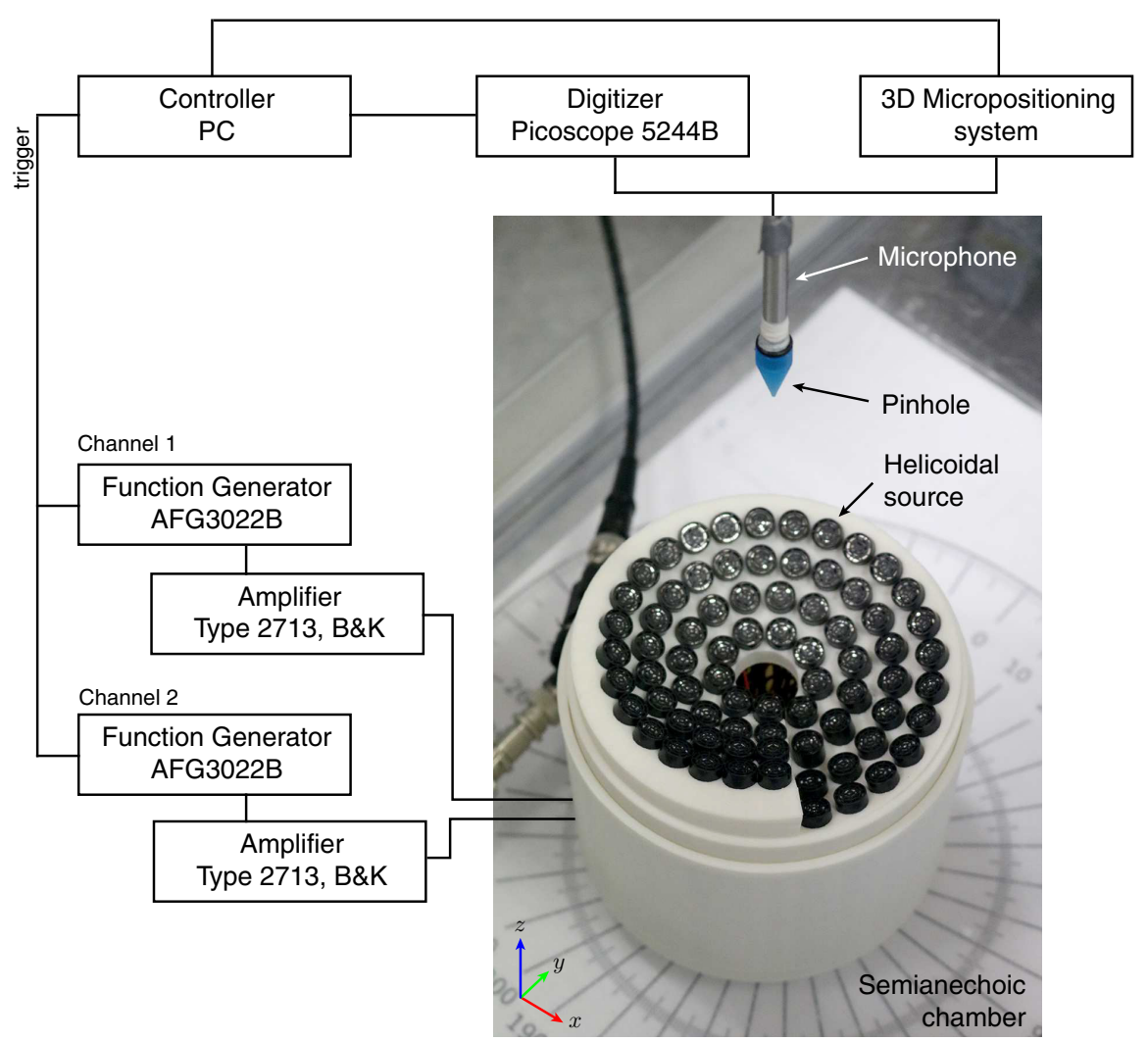

FIG. 6. The experimental setup.

the Rayleigh-Sommerfeld integral, which can be written as

$$
p(\mathbf{r})=-i \frac{\rho_{0} c_{0} k}{2 \pi} \int_{S_{0}} \frac{u_{0}\left(\mathbf{r}_{\mathbf{0}}\right) \exp \left(i k\left|\mathbf{r}-\mathbf{r}_{\mathbf{0}}\right|\right)}{\left|\mathbf{r}-\mathbf{r}_{\mathbf{0}}\right|} d S_{0},
$$

where $S_{0}$ is the radiating surface of the sources located at $\mathbf{r}_{\mathbf{0}}$. Each source vibrates with a particle velocity of $u_{0}\left(\mathbf{r}_{\mathbf{0}}\right)$ normal to its surface $S_{0}$, moving at a frequency $\omega=c_{0} k$, where $k$ is the wave number and $\rho_{0}$ and $c_{0}$ are the density and sound speed of the medium.

\section{Nonlinear regime: Westervelt equation}

The nonlinear field generated by the helical array source is approximated by the $3 \mathrm{D}$ numerical integration of the second-order nonlinear wave equation in a thermoviscous fluid, also known as the Westervelt equation. We use the algorithm described and validated in Ref. [69]. In a retarded frame of coordinates such as $\tau=t-z / c_{0}$, where $t$ is the time and $z$ is the axial coordinate aligned with the beam, the one-way Westervelt equation is written as

$$
\frac{\partial^{2} p}{\partial \tau \partial z}=\frac{c_{0}}{2} \nabla^{2} p+\frac{\beta}{2 \rho_{0} c_{0}^{3}} \frac{\partial^{2} p^{2}}{\partial \tau^{2}}+\frac{b}{2 \rho_{0} c_{0}^{3}} \frac{\partial^{3} p}{\partial \tau^{3}},
$$

where $p$ is the acoustic pressure and $\beta$ is the coefficient of nonlinearity, which for a gas is given by

$$
\beta=\frac{\gamma+1}{2},
$$

where $\gamma=C_{P} / C_{V}$ is the ratio of specific heats or adiabatic index and $b$ the thermoviscous absorption coefficient, given by

$$
b=\zeta+\frac{4}{3} \eta+\kappa\left(\frac{1}{C_{V}}-\frac{1}{C_{P}}\right),
$$

in which $\zeta$ and $\eta$ are the bulk and shear viscosities, $\kappa$ is the thermal conductivity, and $C_{P}$ and $C_{V}$ are the specific heat at constant pressure and volume, respectively.

The numerical integration of Eq. (C2) is sequentially performed along the axial coordinate by using the operator-splitting technique. In the retarded coordinate frame, the field $p(x, y, z, \tau)$ presents a small variation as compared with the field $p(x, y, z+\Delta z, \tau)$ for a small increment in axial coordinate, $\Delta z$. Therefore, we can split Eq. (C2) by its physical processes, obtaining for the diffraction,

$$
\frac{\partial p}{\partial z}=\int \frac{c_{0}}{2} \nabla^{2} p d \tau
$$

for the nonlinearity,

$$
\frac{\partial p}{\partial z}=\frac{\beta}{2 \rho_{0} c_{0}^{3}} \frac{\partial p^{2}}{\partial \tau},
$$

and for the absorption,

$$
\frac{\partial p}{\partial z}=\frac{b}{2 \rho_{0} c_{0}^{3}} \frac{\partial^{2} p}{\partial \tau^{2}} .
$$



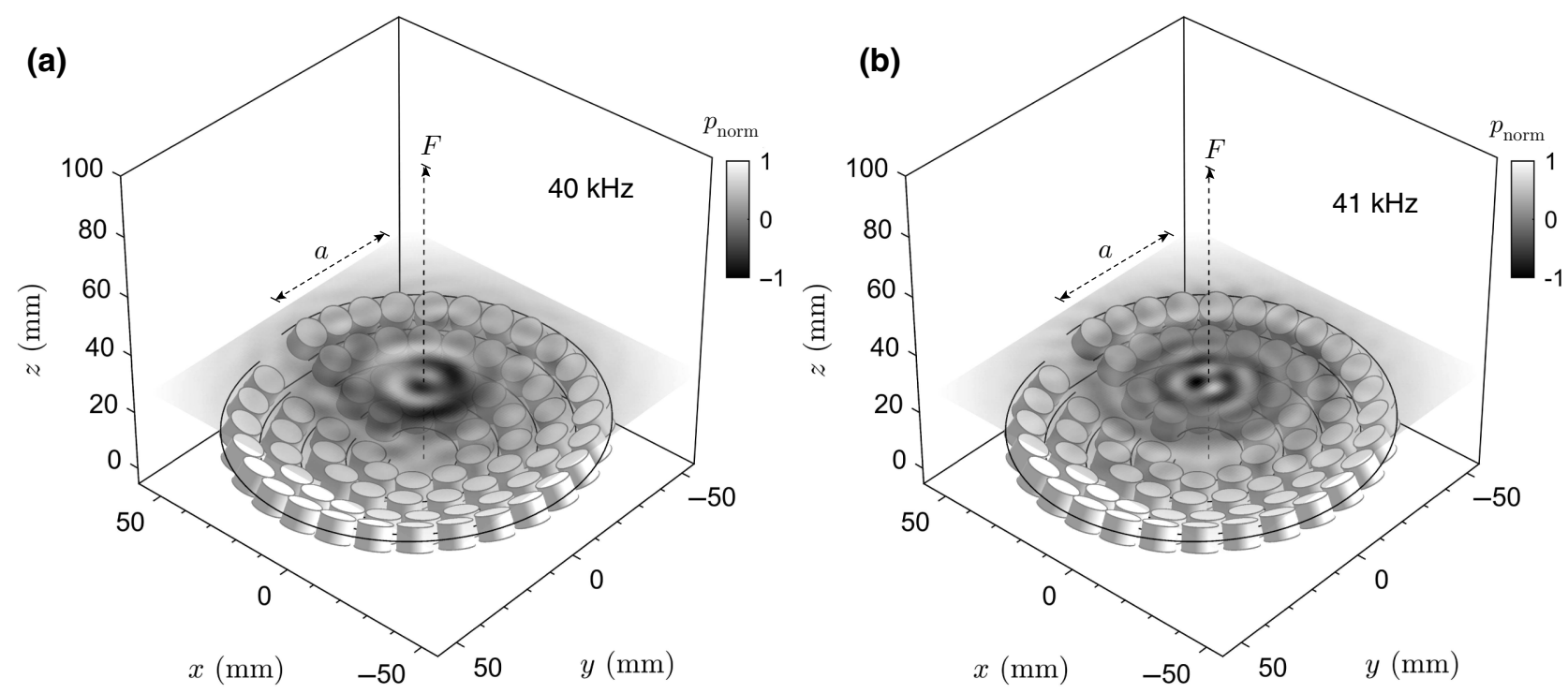

FIG. 7. Near-field integration for the sources using the Rayleigh-Sommerfeld equation to set the initial condition for the nonlinear simulations: (a) first source $\left(l_{1}=1, f_{1}=40 \mathrm{kHz}\right)$; (b) second source $\left(l_{2}=2, f_{2}=41 \mathrm{kHz}\right)$.

The field is expressed in the frequency domain by a finite Fourier-series expansion, including $N$ components as follows:

$$
p(x, y, z, \tau)=\sum_{n=-N}^{n=N} p_{n}(x, y, z) \exp \left(-i \omega_{n} \tau\right),
$$

where $\omega_{n}$ is the frequency of the $n$th spectral component.

Using the operator-splitting technique, the field at a distance $z+\Delta z$ can be obtained as

$$
p(z+\Delta z)=\Gamma_{D}^{[\Delta z / 2]} \Gamma_{N}^{[\Delta z]} \Gamma_{A}^{[\Delta z]} \Gamma_{D}^{[\Delta z / 2]} p(z),
$$

where $\Gamma_{D}^{[\Delta z / 2]}$ approximates the diffraction given by Eq. (C5) over a distance $\Delta z / 2, \Gamma_{N}^{[\Delta z]}$ approximates the nonlinearity given by Eq. (C6) over a distance $\Delta z$, and $\Gamma_{A}^{[\Delta z]}$ approximates the absorption given by Eq. (C7) over a distance $\Delta z$.

The diffraction operator given by $\Gamma_{D}$ is calculated using the angular spectrum method by expanding the field $p_{n}(x, y)$ in the $k$ space $p_{n}\left(k_{x}, k_{y}\right)$, where

$$
\begin{aligned}
& k_{x}=k_{n} \cos (\phi) \sin (\theta), \\
& k_{y}=k_{n} \sin (\phi) \sin (\theta)
\end{aligned}
$$

are the transversal components of the wave vector $k_{n}=$ $\omega_{n} / c_{0}$. Thus, the diffraction operator, applied in the $k$ space, is written as

$$
\Gamma_{D}=\exp \left[i\left(k_{z}-k_{n}\right) \Delta z\right]
$$

with the axial component of the wave number given by

$$
k_{z}=\sqrt{k_{n}^{2}-k_{x}^{2}-k_{y}^{2}} .
$$

As described in Ref. [69], $k$-space filtering and absorbing boundary conditions are used to avoid artificial wave reflections from the boundaries.

The nonlinear operator given by $\Gamma_{N}$ is calculated by expressing Eq. (C6) in the frequency domain for a set of $N$ spectral components as a set of nonlinear coupled equations:

$$
\frac{\partial p_{n}}{\partial z}=\frac{i \beta \omega_{n}}{\rho_{0} c_{0}^{3}}\left(\sum_{k=1}^{N-n} p_{k} p_{n+k}^{*}+\frac{1}{2} \sum_{k=1}^{n-1} p_{k} p_{n-1}\right),
$$

where $p_{n}^{*}$ is the complex conjugate of the harmonic amplitude $p_{n}$. Then, this equation is solved using a fourth-order Runge-Kutta method for a step $\Delta z$.

Finally, assuming an exponentially decaying wave for a small $\Delta z$, the absorption operator is calculated using, for the $n$th spectral component,

$$
\Gamma_{A}=\exp \left(-\frac{b \omega_{n}^{2}}{2 \rho_{0} c_{0}^{3}} \Delta z\right)
$$

The initial conditions for the algorithm are set by integrating the field radiated for each source at a plane located at the exit of the array, $z=F-\sqrt{a^{2}+F^{2}}$, using the Rayleigh-Sommerfeld integral given by Eq. (C1). We use 200 points to mesh the surface of each piezoelectric transducer. The initial conditions are shown in Fig. 7 for the two sources, each one being active at one frequency. Even in the near field, a phase dislocation is already visible at the axis.

For air at a temperature of $T=20^{\circ} \mathrm{C}$, we use the parameters [73] $\rho_{0}=1.21 \mathrm{~kg} / \mathrm{m}^{3}, c_{0}=343 \mathrm{~m} / \mathrm{s}, \gamma=1.4, \zeta=$ $0.6 \eta, \eta=1.85 \times 10^{-5} \mathrm{~Pa} \mathrm{~s}, \kappa=2.59 \times 10^{-2} \mathrm{~W} / \mathrm{mK}$, and 

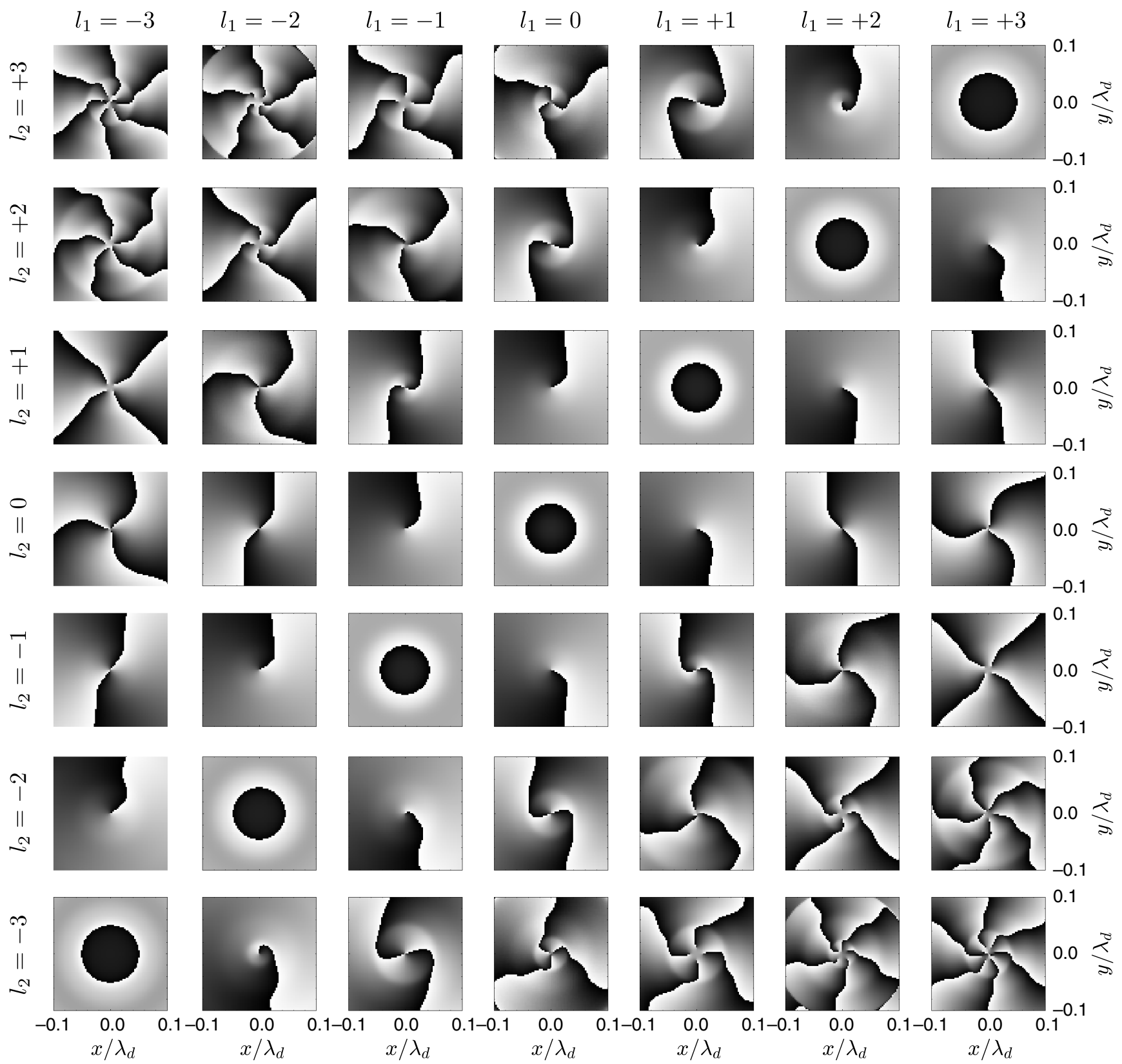

FIG. 8. The phase of the self-demodulated beam as a function of the topological charges of the primary beams.

$C_{P}=1.0061 \times 10^{3} \mathrm{~J} / \mathrm{kg} \mathrm{K}$, leading to $b=4.6 \times 10^{-5} \mathrm{~Pa} \mathrm{~s}$ and $\beta=1.2$. The grid is set to $\Delta z=\lambda_{d} / 240=1.4 \mathrm{~mm}$, the grid steps in the lateral dimensions are $\Delta x=\Delta y=$ $\lambda_{d} / 400=857 \mu \mathrm{m}$, in the domain given by $-2 a<x<2 a$ and $-2 a<y<2 a$, and the number of spectral components is $N=120$, starting at $\omega_{n=1}=\omega_{d}$, i.e., the maximum frequency is $120 \mathrm{kHz}$.

\section{APPENDIX D: CONSERVATION OF TOPOLOGICAL CHARGE IN SELF-DEMODULATED VORTICES}

The phase of the self-demodulated beam depends on the spatiotemporal interference of the two vortex beams, which is linked to their topological charges. In this appendix, we study the conservation of the topological charge by observing the generation of self-demodulated beams with a phase factor $\exp \left(i l_{d} \phi\right)$. Due to the conservation of topological charge of nonlinear vortices, which is a consequence of the conservation of angular momentum, $l_{d}=s_{d}\left(l_{2}-l_{1}\right)$ and $s_{d}=\operatorname{sign}\left(\omega_{2}-\omega_{1}\right)$ if the frequencies of the primary beams are noncommensurate [22,63], which is the common case in parametric acoustic antennas. Figure 8 shows the result of the phase of the self-demodulated beam at the focal spot for $-3,-2, \ldots, l_{1}, \ldots, 2,3$, and $-3,-2, \ldots, l_{2}, \ldots, 2,3$. We can see that the condition $l_{d}=$ $l_{2}-l_{1}$ is fulfilled for all cases, even when the two vortices 
(a)
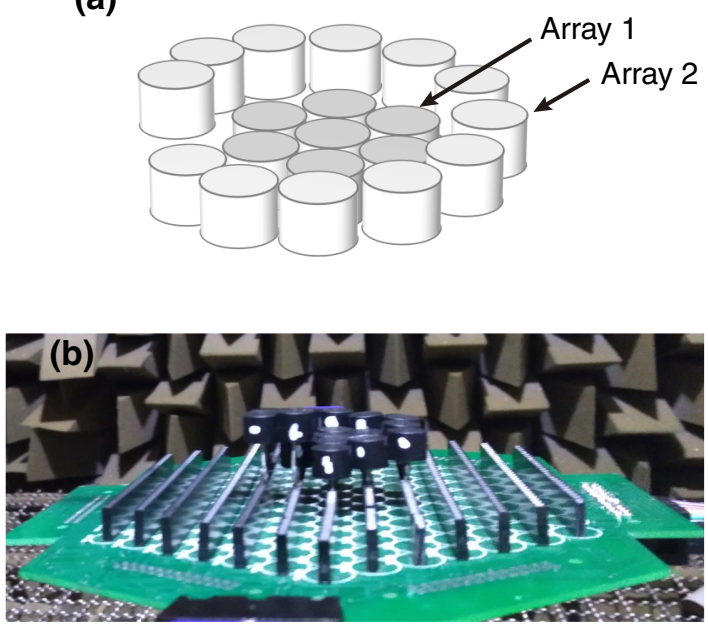
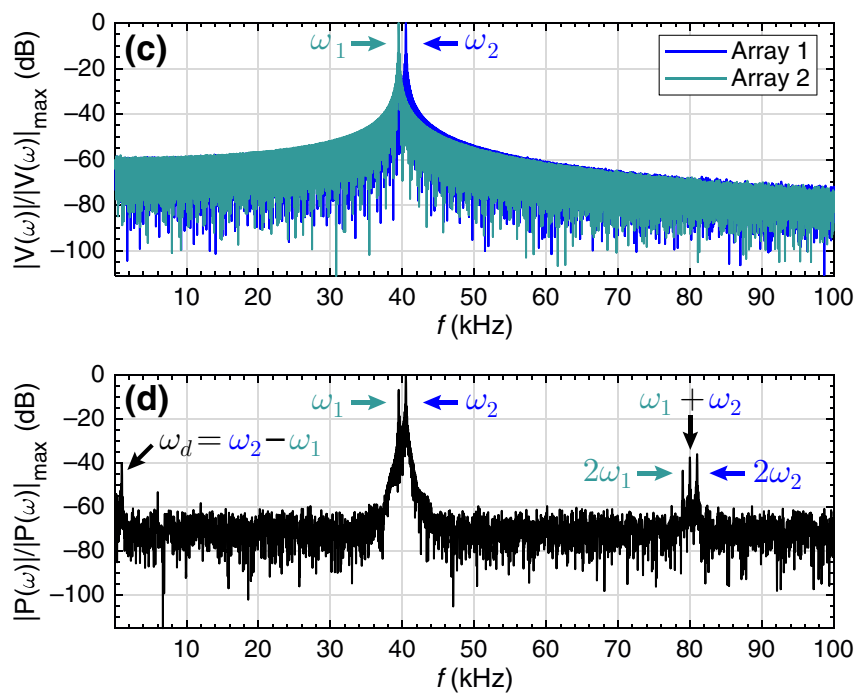

FIG. 9. (a) A schematic of the system, composed of two arrays, to generate divergent waves at frequencies $\omega_{1}$, and $\omega_{2}$. (b) A photograph of the array. (c) The normalized spectrum of the two excitation signals. (d) The normalized spectrum of the acoustic signal captured at the vortex focal spot.

collapse into a self-demodulated beam with no topological charge $\left(l_{1}=l_{2}\right)$.

It can be observed that when ||$l_{1}|-| l_{2}|| \gg 0$, the quality of the synthesized vortex reduces because the field of the two primary beams does not fully overlap at the focal distance. In these cases, by tuning the aperture of each the primary beams, the radial component of the wave vector can be adjusted and, therefore, the width of the primary vortex beams can be adjusted to ensure that they overlap in space. In summary, to synthesize a subwavelength vortex by self-demodulation, the primary beams should present different topological charge and their field distributions must overlap in space.

Another experimental setup is used to experimentally test the conservation of topological charge in selfdemodulated vortices. The source elements of the first array are arranged in a flat circular shape; therefore, $l_{1}=0$. It is composed of $N_{1}=7$ identical transducers (MA40S4S, Murata Manufacturing Co., Ltd., Japan), working at a nominal frequency of $40 \mathrm{kHz}$. The second array consists of a helical surface with $N_{2}=12$ transducers with different heights $h$, as presented in Figs. 9(a) and 9(b). The height of the $n$th source is given by $h(n)=n l_{i} \lambda / N_{i}$, where $N_{i}$ is the total number of transducers and $l_{i}$ the topological charge, $i=1,2$, for the $i$ th array. In our case, we use $l_{2}=1, \lambda=c_{0} / f_{0}=8.6 \mathrm{~mm}$, and $f_{0}=40 \mathrm{kHz}$. Signals are generated using an arbitrary function generator (AFG3022C, Tektronix, Capitol, UK) and an amplifier to drive the two arrays. Each array is excited with a sinusoidal pulse burst of $14 \mathrm{~ms}$ duration. Both arrays are excited with the same amplitude, $5 \mathrm{~V}$. The measurements are conducted in an anechoic chamber. The acoustic signals are acquired using an 1/8-in. calibrated microphone (G.R.A.S.
Holte, Denmark), with a preamplifier and signal conditioning. The signals are acquired using an oscilloscope (GDS2104, Instek, Taipei, Taiwan) with a sample rate of 2 MHz. An $X-Y$ unit stage (Newmark Systems, Rancho Santa Margarita, California) is used to scan the acoustic field. The transverse planes are measured at $z=190 \mathrm{~mm}$ over a squared $X-Y$ plane, $-60 \mathrm{~mm} \leq x \leq 60 \mathrm{~mm}$ and a step of $\Delta x=2.5 \mathrm{~mm}$.

The primary frequencies used for each array are $f_{1}=$ $39.5 \mathrm{kHz}$ and $f_{2}=40.5 \mathrm{kHz}$. The height of the helical source is estimated considering a frequency of $40 \mathrm{kHz}$, equivalent to the nominal frequency of the transducers. With a frequency between 39 and $41 \mathrm{kHz}$, the helical array can generate acoustic vortices with minimal degradation, since the height (pitch) of the source does not change significantly. The difference between the wavelength at 40 $\mathrm{kHz}$ and at $\pm 1 \mathrm{kHz}$ is less than $3 \%$. In this sense, we could excite the helical transducer at a range between $39 \mathrm{kHz}$ and $41 \mathrm{kHz}$.

The spectrum of the input signals is presented in Fig. 9(c), where only two components are observed at frequencies $f_{1}=\omega_{1} / 2 \pi$, and $f_{2}=\omega_{2} / 2 \pi$. On the other hand, the acoustic signal measured at $x=0$ and $y=2.5 \lambda_{1}$ is shown in Fig. 9(d), where besides the input frequencies, the first harmonics can be identified. In addition, the components $\omega_{1}+\omega_{2}$, and $\omega_{d}=\omega_{2}-\omega_{1}$ are presented in the frequency spectrum. The peak at $\omega_{d}$ is of the same order of magnitude as the first harmonics. Conversely, $\omega_{d}$ is 2 orders of magnitude $(-40 \mathrm{~dB})$ lower than the fundamental components.

Two experiments are performed using this setup. For the first experiment, we let the spherical wave front $\left(l_{1}=0\right)$ interfere with the helical wave front $\left(l_{2}=1\right)$, leading to 

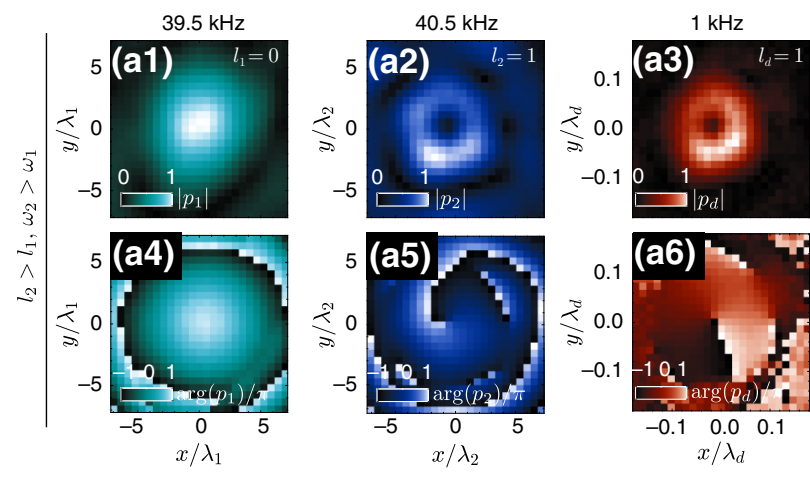

$39.5 \mathrm{kHz}$
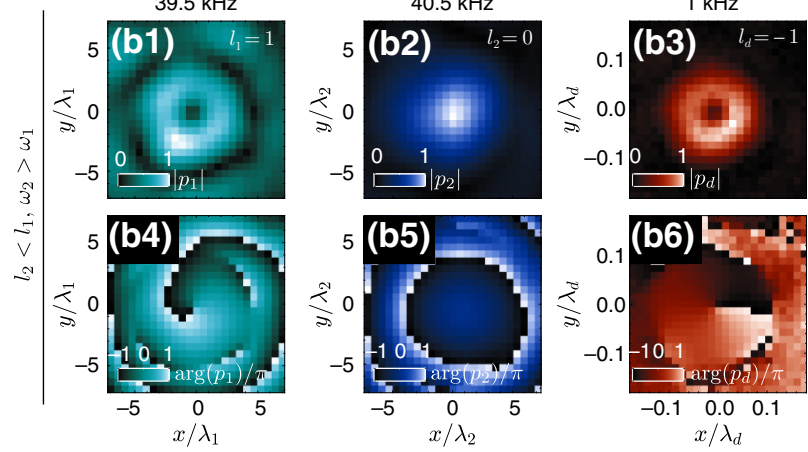

FIG. 10. The (a1),(a2),(b1),(b2) magnitude and (a4),(a5), (b4),(b5) phase of the primary beams and the (a3),(b3) magnitude and (a6),(b6) phase of the self-demodulated vortex beam, obtained experimentally.

a self-demodulated low-frequency helical beam. The selfdemodulated vortex arises as a nonlinear mixing of the two primary waves and due to the conservation of the topological charge. The topological charge of the self-demodulated beam is $l_{d}=l_{2}-l_{1}=1$. Because $l_{2}>l_{1}$, the handedness is clockwise and $l_{d}=l_{2}$, as shown in Figs. 10(a1)-10(a6). The second experiment consists in using the same frequencies but switching the input signals between the two arrays. This is equivalent to $l_{1}>l_{2}$, resulting in $l_{d}=l_{2}-l_{1}=$ -1 . Therefore, the handedness of the self-demodulated vortex is reversed because of the spatiotemporal interference of the two primary vortex beams, as shown in Figs. 10(b1)-10(b6).

One more experiment is carried out using different primary frequencies $f_{1}=[39,39.5,39.7] \mathrm{kHz}$ and $f_{2}=$ $[41,40.5,40.3]$, such that $f_{d}=[2,1,0.6] \mathrm{kHz}$. The results are presented in Fig. 11. As the changes in the frequencies of the primary beams are small, the widths of the primary beams remain almost constant. The magnitude of the field of the self-demodulated beams, presented in Figs. 11(a1)-11(a3), shows ring-shaped structures, the diameters of which remain almost constant.

However, the frequencies of those self-demodulated beams are very different, so the ratios between their widths and their wavelengths are 15.1, 7.8, and 4.6, respectively. Note that these ratios are smaller than in the experiment
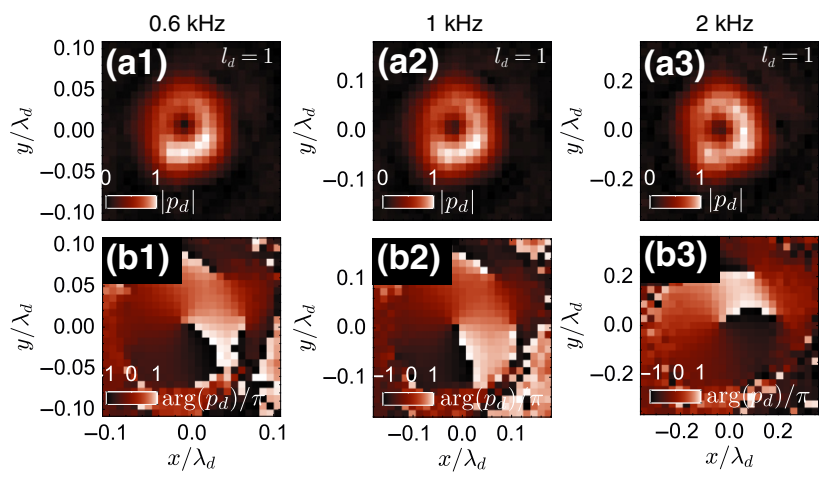

FIG. 11. The experimental results of the acoustic field at $z=$ $19 \mathrm{~mm}$, showing (a1)-(a3) the magnitude and (b1)-(b3) the phase for the self-demodulated vortex at $0.6 \mathrm{kHz}, 1 \mathrm{kHz}$, and $2 \mathrm{kHz}$.

discussed in the main text due to the lack of focusing of the secondary setup. On the other hand, the phase of the field of the self-demodulated vortices is presented in Figs. 11(b1)-11(b3), showing a constant topological charge for all experiments.

[1] W. J. Elias, D. Huss, T. Voss, J. Loomba, M. Khaled, E. Zadicario, R. C. Frysinger, S. A. Sperling, S. Wylie, S. J. Monteith, et al., A pilot study of focused ultrasound thalamotomy for essential tremor, N. Engl. J. Med. 369, 640 (2013).

[2] Y. Tufail, A. Yoshihiro, S. Pati, M. M. Li, and W. J. Tyler, Ultrasonic neuromodulation by brain stimulation with transcranial ultrasound, Nat. Protoc. 6, 1453 (2011).

[3] N. Lipsman, Y. Meng, A. J. Bethune, Y. Huang, B. Lam, M. Masellis, N. Herrmann, C. Heyn, I. Aubert, A. Boutet, et al., Blood-brain barrier opening in Alzheimer's disease using MR-guided focused ultrasound, Nat. Commun. 9, 1 (2018).

[4] J. Nye and M. Berry, in Proc. Math. Phys. Eng. Sci., Vol. 336 (The Royal Society, 1974), Vol. 336, p. 165.

[5] K. Volke-Sepúlveda, A. O. Santillán, and R. R. Boullosa, Transfer of Angular Momentum to Matter from Acoustical Vortices in Free Space, Phys. Rev. Lett. 100, 024302 (2008).

[6] A. Anhäuser, R. Wunenburger, and E. Brasselet, Acoustic Rotational Manipulation Using Orbital Angular Momentum Transfer, Phys. Rev. Lett. 109, 034301 (2012).

[7] C. E. Demore, Z. Yang, A. Volovick, S. Cochran, M. P. MacDonald, and G. C. Spalding, Mechanical Evidence of the Orbital Angular Momentum to Energy Ratio of Vortex Beams, Phys. Rev. Lett. 108, 194301 (2012).

[8] Z. Hong, J. Zhang, and B. W. Drinkwater, Observation of Orbital Angular Momentum Transfer from Bessel-Shaped Acoustic Vortices to Diphasic Liquid-Microparticle Mixtures, Phys. Rev. Lett. 114, 214301 (2015).

[9] P. L. Marston, Radiation force of a helicoidal Bessel beam on a sphere, J. Acous. Soc. Am. 125, 3539 (2009). 
[10] J. Wu, Acoustical tweezers, J. Acous. Soc. Am. 89, 2140 (1991).

[11] D. Baresch, J.-L. Thomas, and R. Marchiano, Observation of a Single-Beam Gradient Force Acoustical Trap for Elastic Particles: Acoustical Tweezers, Phys. Rev. Lett. 116, 024301 (2016).

[12] A. Marzo, M. Caleap, and B. W. Drinkwater, Acoustic Virtual Vortices with Tunable Orbital Angular Momentum for Trapping of Mie Particles, Phys. Rev. Lett. 120, 044301 (2018).

[13] Z. Gong and M. Baudoin, Particle Assembly with Synchronized Acoustic Tweezers, Phys. Rev. Appl. 12, 024045 (2019).

[14] X.-D. Fan and L. Zhang, Trapping Force of Acoustical Bessel Beams on a Sphere and Stable Tractor Beams, Phys. Rev. Appl. 11, 014055 (2019).

[15] C. R. Courtney, B. W. Drinkwater, C. E. Demore, S. Cochran, A. Grinenko, and P. D. Wilcox, Dexterous manipulation of microparticles using Bessel-function acoustic pressure fields, Appl. Phys. Lett. 102, 123508 (2013).

[16] A. Ashkin, Acceleration and Trapping of Particles by Radiation Pressure, Phys. Rev. Lett. 24, 156 (1970).

[17] D. G. Grier, A revolution in optical manipulation, Nature 424, 810 (2003).

[18] M. Baudoin, J.-L. Thomas, R. Al Sahely, J.-C. Gerbedoen, Z. Gong, A. Sivery, O. B. Matar, N. Smagin, P. Favreau, and A. Vlandas, Spatially selective manipulation of cells with single-beam acoustical tweezers, Nat. Commun. 11, 1 (2020).

[19] M. A. Ghanem, A. D. Maxwell, Y.-N. Wang, B. W. Cunitz, V. A. Khokhlova, O. A. Sapozhnikov, and M. R. Bailey, Noninvasive acoustic manipulation of objects in a living body, Proc. Natl. Acad. Sci. U.S.A. 117, 16848 (2020).

[20] D. Baresch and V. Garbin, Acoustic trapping of microbubbles in complex environments and controlled payload release, Proc. Natl. Acad. Sci. U.S.A. 117, 15490 (2020).

[21] B. T. Hefner and P. L. Marston, An acoustical helicoidal wave transducer with applications for the alignment of ultrasonic and underwater systems, J. Acous. Soc. Am. 106, 3313 (1999).

[22] J.-L. Thomas and R. Marchiano, Pseudo Angular Momentum and Topological Charge Conservation for Nonlinear Acoustical Vortices, Phys. Rev. Lett. 91, 244302 (2003).

[23] R. Marchiano and J.-L. Thomas, Synthesis and analysis of linear and nonlinear acoustical vortices, Phys. Rev. E 71, 066616 (2005).

[24] J. F. Pazos-Ospina, J. L. Ealo, and E. E. Franco, Characterization of phased array-steered acoustic vortex beams, J. Acous. Soc. Am. 142, 61 (2017).

[25] J. L. Ealo, J. C. Prieto, and F. Seco, Airborne ultrasonic vortex generation using flexible ferroelectrets, IEEE Trans. Ultrason. Ferroelectr. Freq. Control 58, 1651 (2011).

[26] N. Jiménez, V. Sánchez-Morcillo, R. Picó, L. Garcia-Raffi, V. Romero-Garcia, and K. Staliunas, High-order acoustic bessel beam generation by spiral gratings, Phys. Procedia 70, 245 (2015).

[27] N. Jiménez, R. Picó, V. Sánchez-Morcillo, V. RomeroGarcía, L. M. García-Raffi, and K. Staliunas, Formation of high-order acoustic Bessel beams by spiral diffraction gratings, Phys. Rev. E 94, 053004 (2016).
[28] T. Wang, M. Ke, W. Li, Q. Yang, C. Qiu, and Z. Liu, Particle manipulation with acoustic vortex beam induced by a brass plate with spiral shape structure, Appl. Phys. Lett. 109, 123506 (2016).

[29] N. Jiménez, V. Romero-García, L. M. García-Raffi, F. Camarena, and K. Staliunas, Sharp acoustic vortex focusing by Fresnel-spiral zone plates, Appl. Phys. Lett. 112, 204101 (2018).

[30] X. Jiang, J. Zhao, S.-1. Liu, B. Liang, X.-y. Zou, J. Yang, C.-W. Qiu, and J.-c. Cheng, Broadband and stable acoustic vortex emitter with multi-arm coiling slits, Appl. Phys. Lett. 108, 203501 (2016).

[31] R. D. Muelas-Hurtado, J. L. Ealo, J. F. Pazos-Ospina, and K. Volke-Sepúlveda, Generation of multiple vortex beam by means of active diffraction gratings, Appl. Phys. Lett. 112, 084101 (2018).

[32] R. D. Muelas-Hurtado, J. L. Ealo, and K. Volke-Sepúlveda, Active-spiral Fresnel zone plate with tunable focal length for airborne generation of focused acoustic vortices, Appl. Phys. Lett. 116, 114101 (2020).

[33] A. Riaud, M. Baudoin, O. B. Matar, L. Becerra, and J.-L. Thomas, Selective Manipulation of Microscopic Particles with Precursor Swirling Rayleigh Waves, Phys. Rev. Appl. 7, 024007 (2017).

[34] A. Riaud, J.-L. Thomas, E. Charron, A. Bussonnière, O. B. Matar, and M. Baudoin, Anisotropic Swirling Surface Acoustic Waves from Inverse Filtering for On-Chip Generation of Acoustic Vortices, Phys. Rev. Appl. 4, 034004 (2015).

[35] S. Jiménez-Gambín, N. Jiménez, J. M. Benlloch, and F. Camarena, Generating Bessel beams with broad depth-offield by using phase-only acoustic holograms, Sci. Rep. 9, 1 (2019).

[36] S. Jiménez-Gambín, N. Jiménez, and F. Camarena, Transcranial Focusing of Ultrasonic Vortices by Acoustic Holograms, Phys. Rev. Appl. 14, 054070 (2020).

[37] S. Gspan, A. Meyer, S. Bernet, and M. Ritsch-Marte, Optoacoustic generation of a helicoidal ultrasonic beam, J. Acous. Soc. Am. 115, 1142 (2004).

[38] X. Jiang, Y. Li, B. Liang, J.-c. Cheng, and L. Zhang, Convert Acoustic Resonances to Orbital Angular Momentum, Phys. Rev. Lett. 117, 034301 (2016).

[39] L. Ye, C. Qiu, J. Lu, K. Tang, H. Jia, M. Ke, S. Peng, and Z. Liu, Making sound vortices by metasurfaces, AIP Adv. 6, 085007 (2016).

[40] A. Marzo, A. Ghobrial, L. Cox, M. Caleap, A. Croxford, and B. Drinkwater, Realization of compact tractor beams using acoustic delay-lines, Appl. Phys. Lett. 110, 014102 (2017).

[41] C. J. Naify, C. A. Rohde, T. P. Martin, M. Nicholas, M. D. Guild, and G. J. Orris, Generation of topologically diverse acoustic vortex beams using a compact metamaterial aperture, Appl. Phys. Lett. 108, 223503 (2016).

[42] H. Esfahlani, H. Lissek, and J. R. Mosig, Generation of acoustic helical wavefronts using metasurfaces, Phys. Rev. B 95, 024312 (2017).

[43] J. B. Pendry, Negative Refraction Makes a Perfect Lens, Phys. Rev. Lett. 85, 3966 (2000). 
[44] N. Fang, H. Lee, C. Sun, and X. Zhang, Sub-diffractionlimited optical imaging with a silver superlens, Science 308, 534 (2005).

[45] J. Li, L. Fok, X. Yin, G. Bartal, and X. Zhang, Experimental demonstration of an acoustic magnifying hyperlens, Nat. Mater. 8, 931 (2009).

[46] G. Lerosey, J. De Rosny, A. Tourin, and M. Fink, Focusing beyond the diffraction limit with far-field time reversal, Science 315, 1120 (2007).

[47] C. Shi, M. Dubois, Y. Wang, and X. Zhang, High-speed acoustic communication by multiplexing orbital angular momentum, Proc. Natl. Acad. Sci. U.S.A. 114, 7250 (2017).

[48] X. Jiang, B. Liang, J.-C. Cheng, and C.-W. Qiu, Twisted acoustics: Metasurface-enabled multiplexing and demultiplexing, Adv. Mater. 30, 1800257 (2018).

[49] X. Jiang, C. Shi, Y. Wang, J. Smalley, J. Cheng, and X. Zhang, Nonresonant Metasurface for Fast Decoding in Acoustic Communications, Phys. Rev. Appl. 13, 014014 (2020).

[50] X. Jiang, N. Wang, C. Zhang, X. Fang, S. Li, X. Sun, Y. Li, D. Ta, and W. Wang, Acoustic orbital angular momentum prism for efficient vortex perception, Appl. Phys. Lett. 118, 071901 (2021).

[51] P. J. Westervelt, Parametric acoustic array, J. Acous. Soc. Am. 35, 535 (1963).

[52] H. Zhou, S. Huang, and W. Li, Parametric acoustic array and its application in underwater acoustic engineering, Sensors 20, 2148 (2020).

[53] X.-D. Fan, Z. Zou, and L. Zhang, Acoustic vortices in inhomogeneous media, Phys. Rev. Res. 1, 032014 (2019).

[54] T. Brunet, J.-L. Thomas, and R. Marchiano, Transverse Shift of Helical Beams and Subdiffraction Imaging, Phys. Rev. Lett. 105, 034301 (2010).

[55] L. Li and F. Li, Beating the Rayleigh limit: Orbital-angularmomentum-based super-resolution diffraction tomography, Phys. Rev. E 88, 033205 (2013).

[56] W.-C. Lo, C.-H. Fan, Y.-J. Ho, C.-W. Lin, and C.-K. Yeh, Tornado-inspired acoustic vortex tweezer for trapping and manipulating microbubbles, Proc. Natl. Acad. Sci. 118, e2023188118 (2021).

[57] S. R. Sirsi and M. A. Borden, State-of-the-art materials for ultrasound-triggered drug delivery, Adv. Drug. Deliv. Rev. 72, 3 (2014).

[58] Y. Meng, K. Hynynen, and N. Lipsman, Applications of focused ultrasound in the brain: From thermoablation to drug delivery, Nat. Rev. Neurol. 17, 7 (2021).
[59] I. Basistiy, V. Y. Bazhenov, M. Soskin, and M. V. Vasnetsov, Optics of light beams with screw dislocations, Opt. Commun. 103, 422 (1993).

[60] K. Dholakia, N. Simpson, M. Padgett, and L. Allen, Second-harmonic generation and the orbital angular momentum of light, Phys. Rev. A 54, R3742 (1996).

[61] A. Berzanskis, A. Matijosius, A. Piskarskas, V. Smilgevicius, and A. Stabinis, Conversion of topological charge of optical vortices in a parametric frequency converter, Opt. Commun. 140, 273 (1997).

[62] A. Beržanskis, A. Matijošius, A. Piskarskas, V. Smilgevičius, and A. Stabinis, Sum-frequency mixing of optical vortices in nonlinear crystals, Opt. Commun. 150, 372 (1998).

[63] R. Marchiano and J.-L. Thomas, Doing Arithmetic with Nonlinear Acoustic Vortices, Phys. Rev. Lett. 101, 064301 (2008).

[64] T. Brunet, J.-L. Thomas, R. Marchiano, and F. Coulouvrat, Experimental observation of azimuthal shock waves on nonlinear acoustical vortices, New J. Phys. 11, 013002 (2009).

[65] J.-L. Thomas, T. Brunet, and F. Coulouvrat, Generalization of helicoidal beams for short pulses, Phys. Rev. E 81, 016601 (2010).

[66] For more details about the design of the focused helical source and the experimental setup, see Appendices A and $\mathrm{B}$.

[67] For more details about the conservation of the topological charge of the self-demodulated vortex after the nonlinear mixing process, see Appendix D.

[68] For more details about the analytical model, see Appendix C 1.

[69] P. Yuldashev and V. Khokhlova, Simulation of threedimensional nonlinear fields of ultrasound therapeutic arrays, Acoust. Phys. 57, 334 (2011).

[70] For more details about the numerical method used for the nonlinear simulation of the acoustic vortices, see Appendix C 2.

[71] For additional combinations of topological charges and frequencies, see Appendix D.

[72] M. Baudoin, J.-C. Gerbedoen, A. Riaud, O. B. Matar, N. Smagin, and J.-L. Thomas, Folding a focalized acoustical vortex on a flat holographic transducer: Miniaturized selective acoustical tweezers, Sci. Adv. 5, eaav1967 (2019).

[73] A. D. Pierce, Acoustics: An Introduction to Its Physical Principles and Applications (Springer, Cham, Switzerland, 2019). 